\title{
The outer membrane protease PgtE of Salmonella enterica interferes with the alternative complement pathway by cleaving factors $\mathrm{B}$ and $\mathrm{H}$
}

\author{
Rauna Riva ${ }^{1,2}$, Timo K. Korhonen ${ }^{3}$ and Seppo Meri ${ }^{1,2,4 *}$ \\ ${ }^{1}$ Immunobiology Research Program, Research Program Unit, University of Helsinki, Helsinki, Finland \\ ${ }^{2}$ Department of Bacteriology and Immunology, Haartman Institute, University of Helsinki, Helsinki, Finland \\ ${ }^{3}$ General Microbiology, Department of Biosciences, University of Helsinki, Helsinki, Finland \\ ${ }^{4}$ HUSLAB, Hospital District of Helsinki and Uusimaa, Helsinki, Finland
}

\section{Edited by:}

Amy Rasley, Lawrence Livermore

National Laboratory, USA

Reviewed by:

Miguel A. Valvano, Queen's

University Belfast, UK

Vladimir L. Motin, University of

Texas Medical Branch, USA

\section{${ }^{*}$ Correspondence:}

Seppo Meri, Department of Bacteriology and Immunology, Haartman Institute, University of Helsinki, Haartmaninkatu 3, PO Box 21, Fl-00014 Helsinki, Finland e-mail: seppo.meri@helsinki.fi
The virulence factor PgtE is an outer membrane protease (omptin) of the zoonotic pathogen Salmonella enterica that causes diseases ranging from gastroenteritis to severe enteric fever. It is surface exposed in bacteria that have a short-chain, i.e., rough LPS, as observed e.g., in bacteria residing inside macrophages or just emerging from them. We investigated whether PgtE cleaves the complement factors $B(B)$ and $H$ $(\mathrm{H})$, key proteins controlling formation and inactivation of the complement protein $\mathrm{C} 3 \mathrm{~b}$ and thereby the activity of the complement system. S. enterica serovar Typhimurium or omptin-expressing recombinant $E$. coli bacteria were incubated with purified human complement proteins or recombinant $\mathrm{H}$ fragments. PgtE cleaved both $\mathrm{B}$ and $\mathrm{H}$, whereas its close homolog Pla of Yersinia pestis cleaved only $\mathrm{H}$. $\mathrm{H}$ was cleaved at both $\mathrm{N}$ - and C-termini, while the central region resisted proteolysis. Because of multiple effects of PgtE on complement components (cleavage of $\mathrm{C} 3, \mathrm{C} 3 \mathrm{~b}, \mathrm{~B}$, and $\mathrm{H}$ ) we assessed its effect on the opsonophagocytosis of Salmonella. In human serum, C3 cleavage was dependent on proteolytically active PgtE. Human neutrophils interacted less with serum-opsonized FITC-stained S. enterica 14028R than with the isogenic $\Delta$ pgtE strain, as analyzed by flow cytometry. In conclusion, cleavage of $\mathrm{B}$ and $\mathrm{H}$ by PgtE, together with $\mathrm{C} 3$ cleavage, affects the C3-mediated recognition of $S$. enterica by human neutrophils, thus thwarting the immune protection against Salmonella.

Keywords: Salmonella, protease, phagocytosis, immune evasion, PgtE, complement factor B, complement factor H, omptin

\section{INTRODUCTION}

Salmonella enterica serovar Typhi causes typhoid fever, a systemic disease with a global annual burden of 27 million cases and a case-fatality rate of $1 \%$. In contrast to the human-restricted Typhi, most S. enterica serovars are zoonotic pathogens. Indeed, salmonellosis is the second most common zoonosis in Europe, after Campylobacter infections. While in humans the serovar Typhimurium causes mainly a self-limited gastroenteritis, in susceptible mouse strains it causes a disease similar to human typhoid fever. It can cause a systemic disease also in humans. Notably, it is the leading serotype causing invasive non-typhoidal salmonellosis in Africa (Crump et al., 2004; Buckle et al., 2010; Graham, 2010; Wick, 2011; Eurosurveillance Editorial Team, 2012).

The complement system is in the front line of immune defense against invading microbes. It comprises $\sim 50$ proteins present in plasma or on cell surfaces. A contact with bacteria activates a

Abbreviations: AP, alternative complement pathway; B, factor B; $\mathrm{H}$, factor $\mathrm{H}$; $\mathrm{HIS}$ heat-inactivated NHS; NHS, normal human serum pool; pAb, polyclonal antibody; SCR, short consensus repeat; VBS, veronal-buffered saline cascade of serine protease reactions initiated by the alternative (AP), classical or lectin pathway. These pathways converge when C3 is activated by cleavage into C3a and C3b. C3b can bind covalently onto the bacterial surface, where it promotes phagocytosis as a key opsonin. Importantly, it nucleates the exponential amplification of complement cascade via AP. C3b can associate with the pro-enzyme factor B (B) that gets proteolytically activated by factor $\mathrm{D}$ into active $\mathrm{Bb}$. The $\mathrm{C} 3 \mathrm{bBb}$ complex, i.e., $\mathrm{AP}$ C3-convertase, generates more $\mathrm{C} 3 \mathrm{~b}$ molecules by liberating the anaphylatoxic peptide $\mathrm{C} 3 \mathrm{a}$ from the $\mathrm{C} 3 \alpha$ chain of $\mathrm{C} 3$. Ultimately, complement activation leads to the formation of membrane attack complex that disrupts the outer membranes of Gramnegative bacteria to induce osmotic lysis (Torreira et al., 2009; Ricklin et al., 2010). The self-amplifying property of complement warrants tight regulation to avoid excessive inflammation and damage to bystander host cells. Factor $\mathrm{H}(\mathrm{H})$ is a singlechain $\sim 155 \mathrm{kDa}$ glycoprotein that consists of 20 homologs short consensus repeat (SCR) domains, aka complement control protein (CCP) domains. Factor $\mathrm{H}$ regulates complement activity by three means. It is the main soluble co-factor for factor I-mediated inactivation of $\mathrm{C} 3 \mathrm{~b}$ into $\mathrm{iC} 3 \mathrm{~b}$ that is the preferred ligand for the 
phagocytic CR3 (CD11b/CD18) receptor. Factor $\mathrm{H}$ also inhibits the assembly and accelerates the decay of the AP C3-convertase C3bBb (Bajic et al., 2013; Makou et al., 2013). Many pathogens recruit $\mathrm{H}$ onto their surfaces to inhibit complement attack (Blom et al., 2009; Meri et al., 2013).

PgtE of S. enterica and Pla of Yersinia pestis that causes plague, belong to the family of enterobacterial outer membrane proteases called omptins. Omptins are structurally homologs $\sim 70 \AA$ $\beta$-barrel-folded transmembrane proteins exposing five loops on the bacterial surface. The omptins have a highly conserved catalytic groove and they cleave substrates after basic amino acids. However, the amino acid sequences as well as the lengths of the surface loops in omptins from different bacterial species are variable and dictate the polypeptide substrate specificity of individual omptins (Kukkonen et al., 2001; Ramu et al., 2008; Haiko et al., 2010; Korhonen et al., 2013). The omptins are unique surface proteases as they require short-chain, i.e., rough, LPS for their enzymatic activity toward polypeptide substrates (Kukkonen et al., 2004; Lahteenmaki et al., 2005; Eren et al., 2010; Eren and van den Berg, 2012). Salmonella is a facultatively intracellular bacterium residing mainly in permissive macrophages, where it expresses a shortened O-antigen and active PgtE (Lahteenmaki et al., 2005). $\mathrm{Pla}$ is active on the surface of $Y$. pestis, which inherently has a rough LPS type due to frameshift mutations within the $\mathrm{O}$-antigen biosynthesis gene cluster (Skurnik et al., 2000). Also, Y. pestis modifies its LPS structure upon transfer from the flea temperature to the human temperature to favor enzymatic activity of Pla (Suomalainen et al., 2010) and to suppress innate immune responses to LPS (Montminy et al., 2006). In contrast to Y. pestis, S. enterica mainly spreads within the host as an intracellular pathogen that expresses rough LPS during its growth within or immediately after release from phagocytes (Eriksson et al., 2003; Lahteenmaki et al., 2005). In vivo studies in mouse models have established that both PgtE and Pla contribute to pathogenesis. Deletion of $p g t E$ reduces the growth in macrophages and the systemic dissemination of Salmonella Typhimurium into liver and spleen by tenfold after intraperitoneal inoculation (Lahteenmaki et al., 2005; Ramu et al., 2008). Pla is central for the establishment of pneumonic and bubonic plague, and deletion of the pla gene attenuates $Y$. pestis in bubonic plague by millionfold (Sodeinde et al., 1992; Sebbane et al., 2006; Lathem et al., 2007).

Several complement proteins are omptin substrates. We previously reported that PgtE cleaves C3, C3b, C4, C4b, and C5 (Ramu et al., 2007). Pla cleaves C3 but is inactive against factor B (Sodeinde et al., 1992). Cpa, the omptin of the invasive pathogen Cronobacter sakazakii, cleaves C3, C3a, and C4b (Franco et al., 2011). Haiko and co-workers reported that PgtE and Pla cleave vitronectin that inhibits the membrane attack complex (Podack and Muller-Eberhard, 1979; Haiko et al., 2010). Here we investigated, whether the central complement factors $\mathrm{B}$ and $\mathrm{H}$ could be cleaved by PgtE and selected omptins of Yersinia and E. coli. Furthermore, since human serum contains several PgtE substrates and since the aforementioned studies have been conducted with purified complement proteins, we deemed it crucial to confirm the PgtE-mediated degradation of C3 in human serum. Finally, since factors $\mathrm{B}$ and $\mathrm{H}$ have opposing effects on the formation of $\mathrm{C} 3 \mathrm{~b}$, the key opsonin, it was important to test how PgtE affects the opsonophagocytic uptake of Salmonella into human neutrophils.

\section{RESULTS \\ CLEAVAGE OF FACTORS B AND H BY SALMONELLA IS PgtE-DEPENDENT}

We first analyzed whether the virulent strain 14028 of S. enterica has the capacity to cleave the complement proteins $\mathrm{B}$ and $\mathrm{H}$. Factor B is central to complement activity as factor D-activated $\mathrm{Bb}$ is the protease part of the $\mathrm{C} 3$-convertases $\mathrm{C} 3\left(\mathrm{H}_{2} \mathrm{O}\right) \mathrm{Bb}$ and C3bBb (Torreira et al., 2009). B contains SCR-modules homologs to those in the regulatory factor $\mathrm{H}$, and it has an opposite, complement activation promoting effect (Milder et al., 2007). For two reasons, the degradation of factors $\mathrm{B}$ and $\mathrm{H}$ was analyzed using the rough variant $14028 \mathrm{R}$ that is resistant to a smooth LPSspecific bacteriophage P22c2 (Wick et al., 1994). Enterobacterial omptins require rough LPS for enzymatic activity, and O-chains, which are typically long in in vitro-grown Typhimurium, sterically inhibit proteolysis (Kukkonen et al., 2004). S. Typhimurium 14028 modifies its LPS into a rough form during infection. Both the activity and expression levels of the PgtE protease of 14028 are high in cells isolated from macrophages (Eriksson et al., 2003; Lahteenmaki et al., 2005). Thus, the rough strain 14028R grown in pgtE-inducing medium mimics phagocytereleased S. enterica. The strain 14028R cleaved both B and H, whereas the $\Delta p g t E$ derivative 14028R-1 did not exhibit these activities (Figure 1). Complementation of 14028R-1 with pgtEencoding pMRK 3 recovered the activity toward $\mathrm{B}$ and $\mathrm{H}$, whereas the introduction of the empty vector plasmid had no effect. Thus, PgtE was required for the cleavage of $\mathrm{B}$ and $\mathrm{H}$ by Salmonella.

To confirm the role of PgtE in the degradations, we expressed $p g t E$ in E. coli 83972 $\Delta o m p T$, which is deleted for the endogenous omptin gene ompT and lacks ompP (Järvinen et al., 2013). E. coli 83972 $\Delta$ ompT expressing PgtE cleaved B in a time-dependent fashion generating four immunoreactive fragments (Figure 2A). In non-reducing conditions the apparent sizes of the major fragments corresponded to those of the fragments $\mathrm{Bb}$ and $\mathrm{Ba}$ generated by factor D. PgtE generated also B fragments of approximately 57 and $28 \mathrm{kDa}$. However, when the samples were run under reducing conditions (Figures 2B,C) the PgtE-generated cleavage pattern of factor $\mathrm{B}$ was different. The Ba-sized or the $28 \mathrm{kDa}$-sized fragment could not be detected indicating cleavages at multiple sites. The different cleavage patterns are apparently due to intramolecular disulfide bridges that keep the proteolytically cleaved fragments together under non-reducing conditions. No other omptins, examined as controls, were able to cleave factor B (Figure 2C).

E. coli 83972 $\Delta$ ompT (Figure 3A) and E. coli XL1 (Figure 3B) expressing PgtE cleaved factor $\mathrm{H}$ at multiple sites resulting in ladder-like cleavage patterns. In comparison, $Y$. pestis Pla cleaved factor $\mathrm{H}$ less efficiently compared to PgtE, since more intact protein was left after incubation with the Pla-expressing strains. Previous reports have identified Asp206 as a catalytic amino acid in omptins (Kukkonen et al., 2001; Lahteenmaki et al., 2005; Eren et al., 2010; Haiko et al., 2010; Järvinen et al., 2013). E. coli XL1 bacteria expressing the catalytic-site mutated PgtE D206A did not cleave factors B (Figure 2B) or H (Figure 3B). The catalytic-site 


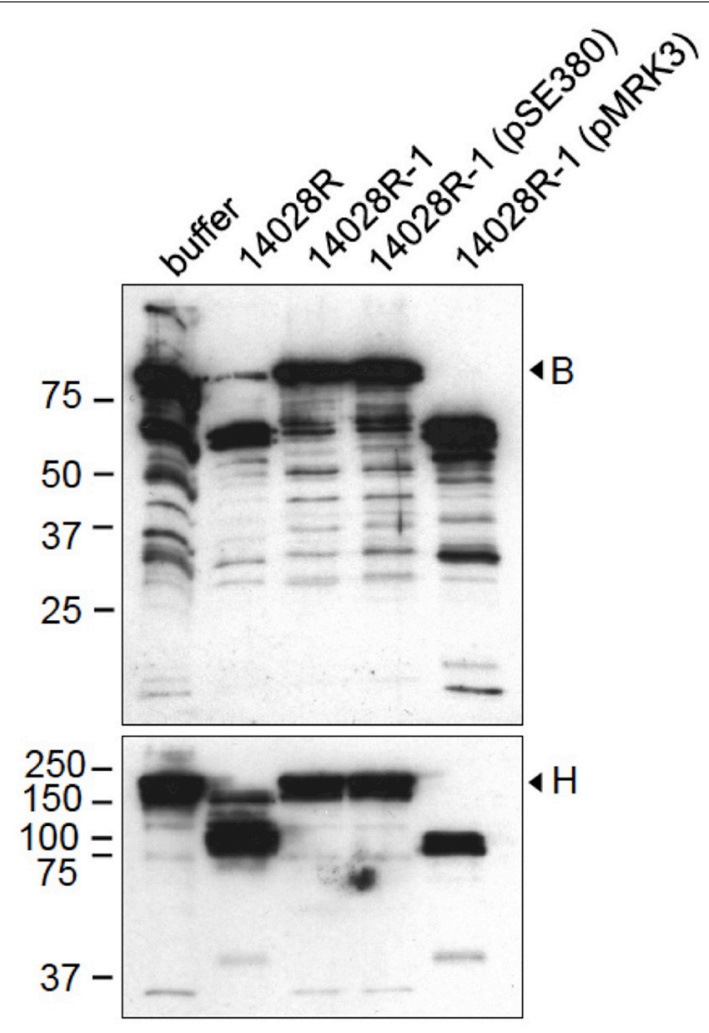

FIGURE 1 | Cleavage of factors $B$ and $H$ by $S$. enterica is

PgtE-dependent. S. enterica 14028R, its $\Delta$ pgtE-derivative 14028R-1 and PgtE (pMRK3)- or empty vector (pSE380) -complemented 14028R-1 strains were incubated with factors $\mathrm{B}$ or $\mathrm{H}(33 \mu \mathrm{g} / \mathrm{ml})$ for $4 \mathrm{~h}$. The reduced supernatants were immunoblotted with polyclonal antibodies against factors $\mathrm{B}$ or $\mathrm{H}$. The assays were conducted at least twice with consistent results.

substitution D206A rendered also Pla inactive against factor $\mathrm{H}$ (Figure 3B). Thus, the breakdown of factors B and $\mathrm{H}$ was critically dependent on the direct proteolytic activity exerted by these omptins.

\section{MAPPING OF THE CLEAVAGE SITES IN H}

PgtE and Pla cleaved $\mathrm{H}$ into ladder-like patterns indicating cleavages at several sites (Figures 3A,B). H consists of 20 consecutive globular SCR domains connected by short linkers (Makou et al., 2013). To approximate the cleavage sites in H, E. coli $83972 \Delta$ ompT expressing PgtE or Pla were incubated with recombinant, C-terminally His-tagged fragments of $\mathrm{H}$. The areas of $\mathrm{H}$ covered by the recombinant fragments SCR1-5 and SCR15-20 are illustrated in Figure 4A. PgtE cleaved the N-terminal fragment SCR1-5 completely during a 1-h incubation, since the epitopes for both the Penta-His mAb and the polyclonal anti-H antibody $(\mathrm{H} \mathrm{pAb})$ disappeared. In contrast, Pla did not cleave SCR1-5 (Figure 4B).

PgtE cleaved also the SCR15-20 fragment representing the C-terminus of $\mathrm{H}$, since the intact SCR15-20 detected with either the Penta-His $\mathrm{mAb}$ or $\mathrm{H}$ pAb disappeared. The $\mathrm{H}$ pAb recognized cleavage fragments of $\sim 25 \mathrm{kDa}$ and below indicating

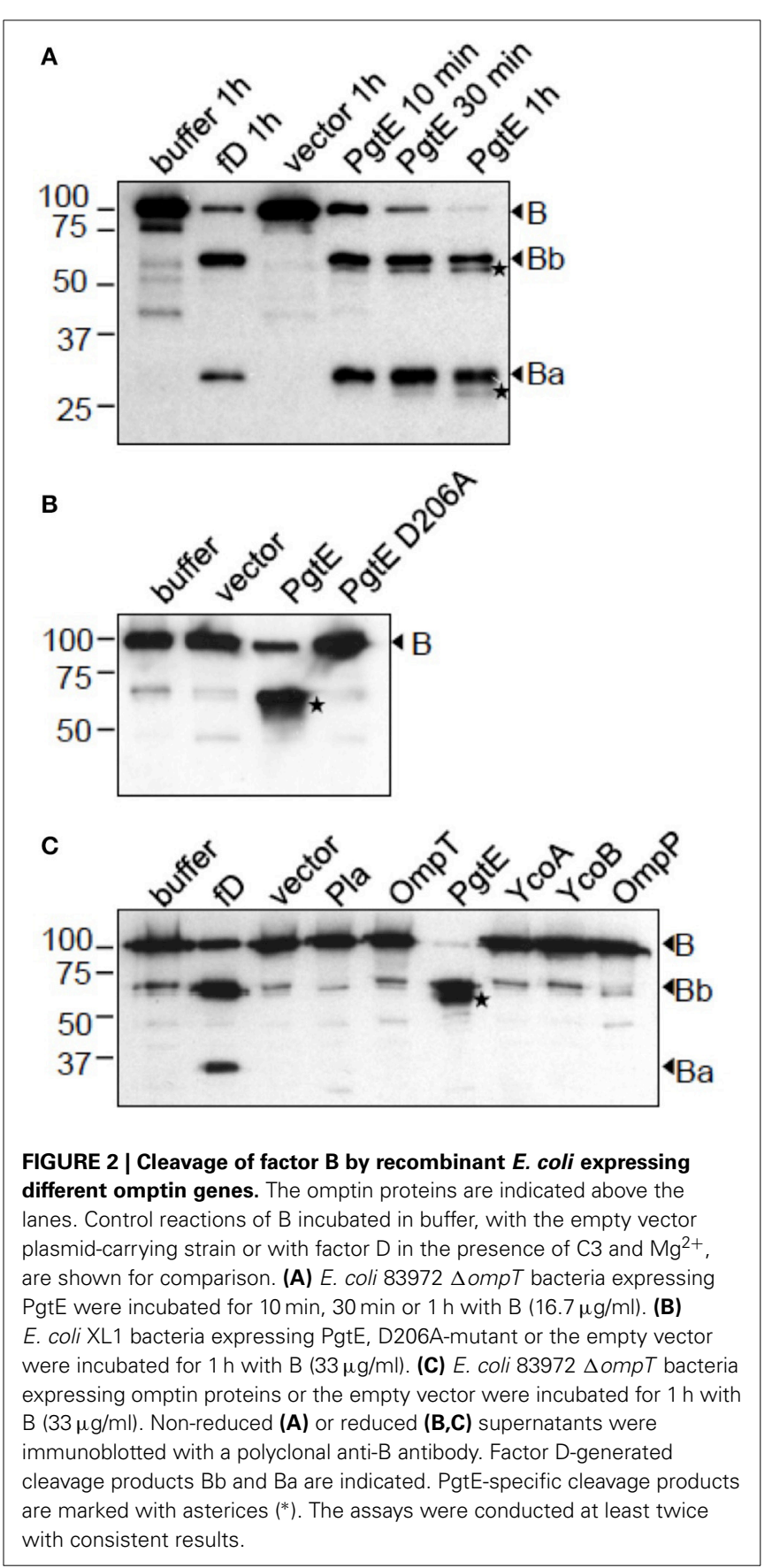

several cleavage sites. Pla cleaved SCR15-20 approximately in the middle of the construct and close to either terminus since $\mathrm{H} \mathrm{pAb}$ recognized a fragment slightly smaller than the intact SCR15-20 and a fragment of $\sim 25 \mathrm{kDa}$ covering $\sim 3$ SCRs (Figure 4C). The $\mathrm{H}$ pAb, but not Penta-His $\mathrm{mAb}$, recognized also an apparently intact-sized fragment. This could be explained if Pla cleaves off the C-terminal His-tail after SCR20, resulting in the loss of the Penta-His mAb epitope (Kühn and Zipfel, 1995). The amino acid sequence of human factor $\mathrm{H}$ terminates in Arg that is a potential P1 residue for omptins (Hritonenko and Stathopoulos, 2007).

Next we continued the cleavage site mapping with purified factor $\mathrm{H}$ and monoclonal antibodies targeting different epitopes 
along $\mathrm{H}$. The mAb 196X recognizes the N-terminal SCR1, the 131X recognition site lies within SCRs 8 through 14.5 and 3D11 recognizes SCR18 as illustrated in Figure 4A. PgtE cleaved $\mathrm{H}$ near both termini (Figure 4D) supporting the results with the recombinant SCR1-5 and SCR15-20 fragments (Figures 4B,C),

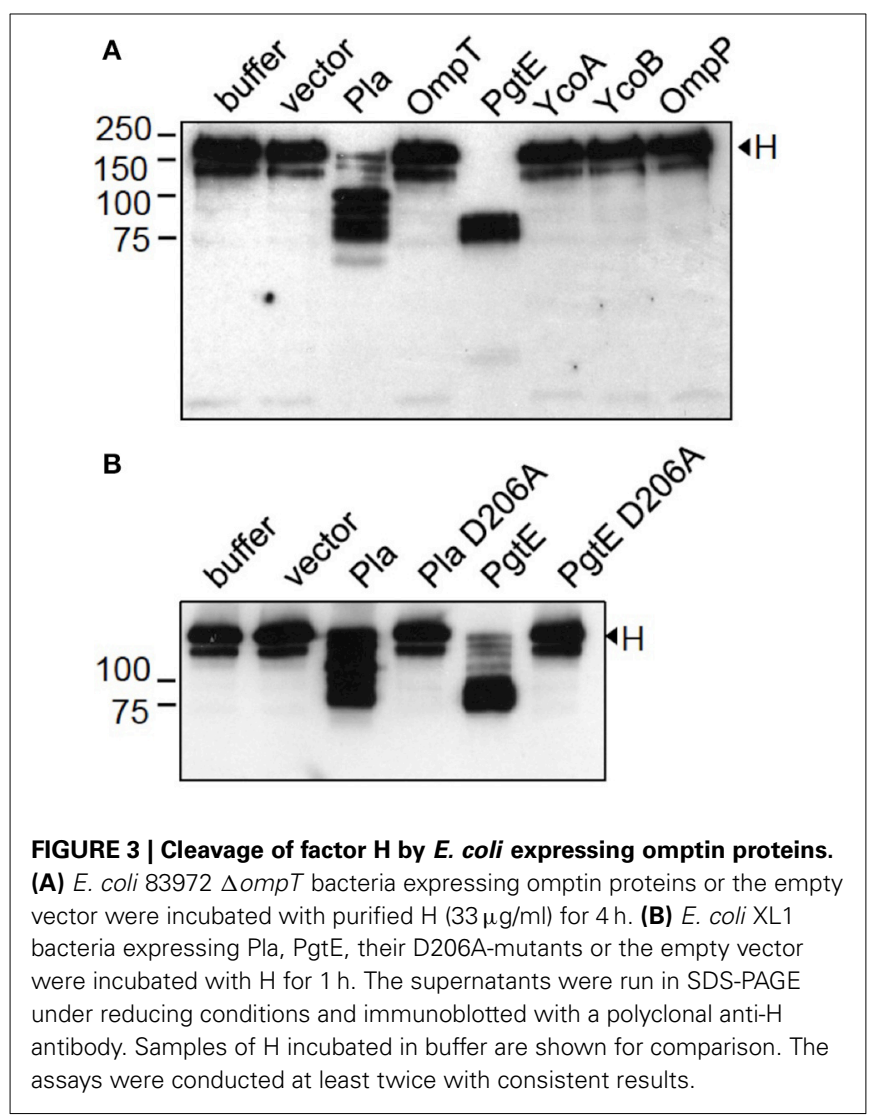

since both the 196X epitope at SCR1 and the 3D11 epitope at SCR18 disappeared. The 131X and pAb recognized an $\sim 85 \mathrm{kDa}$ fragment that represents the central region of $\mathrm{H}$ covering approximately 11 SCRs, using the rough estimate of $7.8 \mathrm{kDa}$ per SCR. E. coli 83972 $\Delta$ ompT expressing Pla cleaved factor $\mathrm{H}$ incompletely in one $\mathrm{h}$ since all antibodies recognized the intact $\mathrm{H}$. The antibodies additionally recognized one (196X) or two (131X, 3D11, pAb) smaller cleavage products. The $120 \mathrm{kDa} H$ fragment recognized by 196X, 131X and pAb represented approximately 15.5 of the most N-terminal SCRs. The $112 \mathrm{kDa}$ fragment recognized by $131 \mathrm{X}, 3 \mathrm{D} 11$, and pAb represented approximately 14.5 of the most C-terminal SCRs. This indicated that Pla left the centermost SCRs (approximately 5.5 through 15.5) intact. The cleavage sites were most probably located within linker regions connecting the SCR domains since the non-reduced samples, in which the intraSCR disulphide bonds remain intact, migrated as separate bands. In conclusion, Pla cleaves shortly after SCR5 and also nearer the C-terminus of $\mathrm{H}$, after SCR15.

\section{PgtE INHIBITS OPSONIZATION OF S. ENTERICA 14028R}

We have previously reported that PgtE cleaves purified C3 and C3b (Ramu et al., 2007). However, the cleavage of factors B and $\mathrm{H}$ by PgtE could affect the fate of C3 in serum. Firstly, B and $\mathrm{H}$ could compete with $\mathrm{C} 3 / \mathrm{C} 3 \mathrm{~b}$ as substrates for cleavage by PgtE. Secondly, cleavage of $\mathrm{H}$ could abolish its complement regulatory activity leading to increased formation of $\mathrm{C} 3 \mathrm{~b}$, whereas cleavage of B could lead to fewer active $\mathrm{C} 3$ convertases and decreased formation of C3b. Thirdly, PgtE could compete with B and $\mathrm{H}$ for $\mathrm{C} 3 / \mathrm{C} 3 \mathrm{~b}$ cleavage, because $\mathrm{Bb}$ in the $\mathrm{C} 3$-convertase cleaves $\mathrm{C} 3$, and $\mathrm{H}$ is a cofactor for $\mathrm{C} 3 \mathrm{~b}$ cleavage by factor I (Ramu et al., 2007; Torreira et al., 2009; Makou et al., 2013). To test the net effect of PgtE on C3 in the presence of all these factors Salmonella strains were incubated in 5\% NHS. This serum concentration was chosen to allow an appropriate ratio between the
A

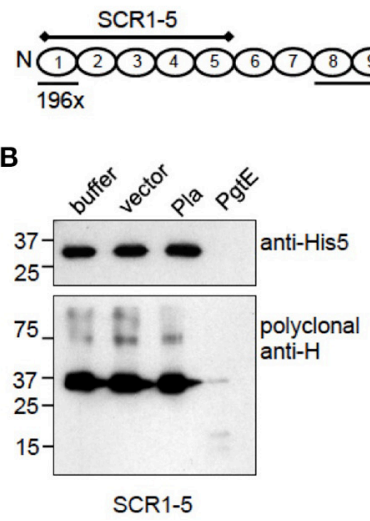

D

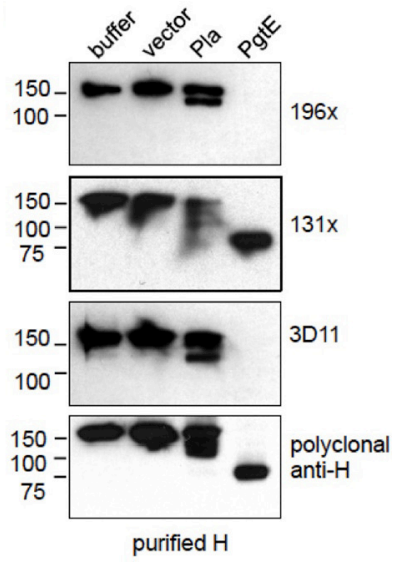

FIGURE 4 | Mapping of cleavage sites in factor $\mathbf{H}$. (A) Graphic of factor $\mathrm{H}$ indicating the areas covered by the recombinant fragments SCR1-5 and SCR15-20 and the localization of the 196X, 131X, and 3D11 mAb epitopes. (B-D) E. coli $83972 \triangle o m p T$ bacteria expressing PgtE, Pla or the empty vector were incubated for $1 \mathrm{~h}$ with equimolar amounts of recombinant $\mathrm{H}$ fragments SCR1-5, SCR15-20 or purified $\mathrm{H}$. The non-reduced supernatants

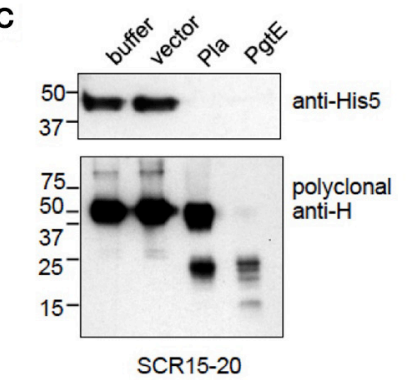

were run in SDS-PAGE and immunoblotted. (B) SCR1-5 and (C) SCR15-20 blotted with a monoclonal anti-His5 Ab recognizing the C-terminal His8-tag in the $\mathrm{H}$ fragments, and with a polyclonal anti-H Ab. (D) Purified $\mathrm{H}$ blotted with monoclonal Abs 196X, 131X, and 3D11 recognizing the N-terminal SCR1, the SCRs 8-14.5 and the SCR18, respectively; or with a polyclonal anti-H Ab. The assays were conducted at least twice with consistent results. 
enzyme (PgtE) and the substrates $(\mathrm{B}, \mathrm{H})$ for the catalytic reaction. In the human body the bacteria would encounter a whole range of concentrations of $\mathrm{B}$ and $\mathrm{H}$ because of their diffusion from blood plasma. C $3 \alpha$ chain was cleaved into two fragments of $\sim 63$ and $\sim 38 \mathrm{kDa}$ when incubated with 14028R (Figure 5A). Notably, the $\sim 63 \mathrm{kDa}$ fragment was not directly PgtE-generated, since PgtE cleaves C $3 \alpha / \alpha^{\prime}$ into fragments of $46 \mathrm{kDa}$ and smaller (Ramu et al., 2007, and data not shown). Neither does the inactivation of C3b by factor I or plasmin result in a $63 \mathrm{kDa}$ band. However, albumin can affect the migration of nearby bands, thus the $\sim 63 \mathrm{kDa}$ band could be the $68 \mathrm{kDa}$ fragment produced by factor I or plasmin (Barthel et al., 2012)14028R-1 (pMRK3) overexpressing PgtE completely depleted the intact $\mathrm{C} 3 \alpha$. In contrast, vector- or PgtE D206A-complemented strains did not affect C3 $\alpha$ indicating that $\mathrm{C} 3 \alpha$ depletion was dependent on proteolytically active PgtE (Figure 5B). To rule out the possibility that PgtEexpressing bacteria bound C3 fragments and pulled them down, the pellets were examined, too. 14028R-1 (pMRK3) had actually less C3-derived fragments on its surface compared to the vectorcarrying strain 14028R-1(pSE380), or to 14028R-1 (pMRK31) expressing PgtE D206A. This indicated that in the absence of proteolytically active PgtE complement activation was directed onto the Salmonella surface. Also the activity of PgtE in the presence of all other serum proteins was thus confirmed.

\section{PgtE INTERFERES WITH OPSONOPHAGOCYTOSIS OF SALMONELLA}

Since opsonization by $\mathrm{C} 3 \mathrm{~b}$ or $\mathrm{iC} 3 \mathrm{~b}$ enhances the uptake of microbes by phagocytes (Ricklin et al., 2010; Bajic et al., 2013), we tested whether PgtE-mediated depletion of C3 from serum would affect phagocytosis. Our experimental setting analyzed collectively both internalized and adherent bacteria. As shown in Figure 6A the fluorescence signal from human PMN incubated with FITC-stained, NHS-opsonized 14028R for $10 \mathrm{~min}$ was weaker than that from PMN incubated with pgtE-negative 14028R-1. This was not the case when the strains were mocktreated with buffer or HIS suggesting that the effect seen with NHS was dependent on active complement and not on antibodies or direct contact between bacteria and PMN. In conclusion, the expression of PgtE impaired complement-mediated phagocytosis of 14028 R.

\section{DISCUSSION}

Microbial proteins that mediate resistance to serum killing or to phagocytosis often recruit complement regulators, such as factor $\mathrm{H}$, onto the microbial surface (Blom et al., 2009; Meri et al., 2013). The proteolytic cleavage of factor B by the virulence factor PgtE of S. enterica shown in this study highlights another mechanism of invasive bacteria to resist complement-mediated defense. We also

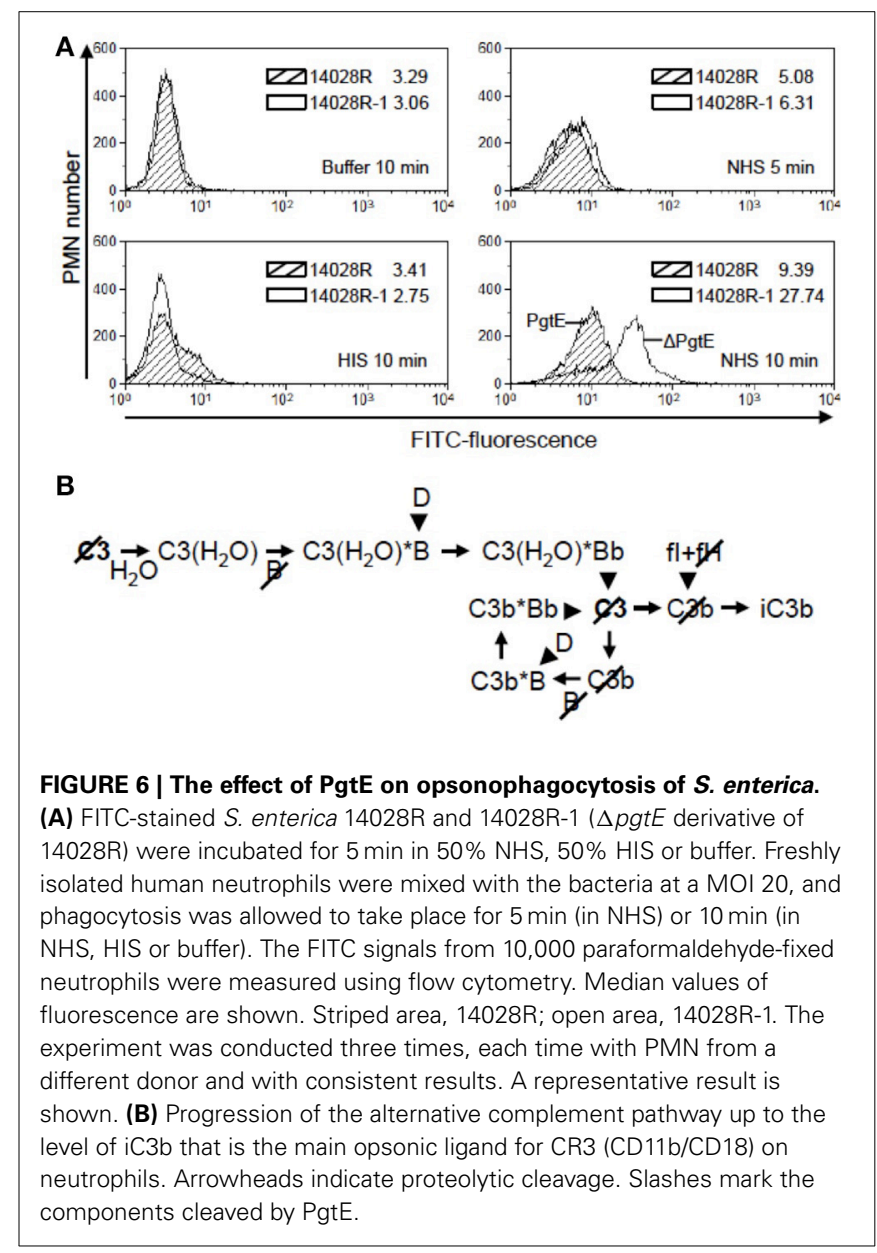

A

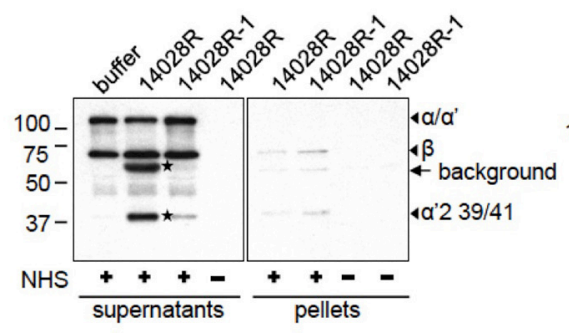

FIGURE 5 | C3 inactivation by $\boldsymbol{S}$. enterica in serum. (A) S. enterica 14028R and 14028R-1 or (B) 14028R-1 strains complemented with PgtE (pMRK3), PgtE D206A (pMRK31) or empty vector (pSE380), were incubated in $5 \%$ NHS-VBS or in plain VBS for $1 \mathrm{~h}$ and pelleted. The

B

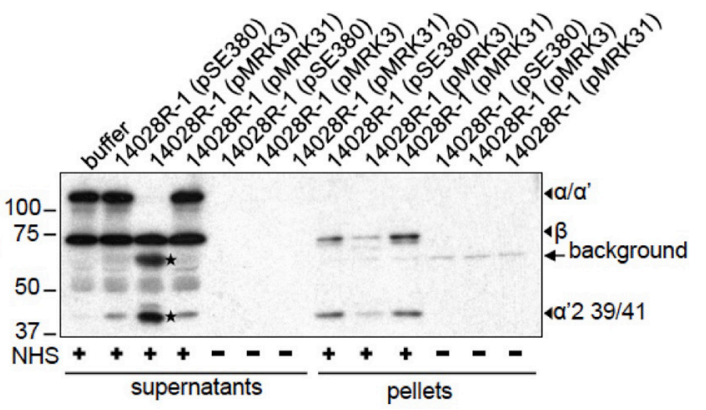

reduced supernatants and washed pellets were immunoblotted with anti-C3c and -C3d antibodies. The cleavage fragments of C3 and the non-specific signal from bacteria-originating epitopes are indicated. PgtE-mediated cleavages are marked with asterices $(*)$. 
observed cleavage of factor $\mathrm{H}$, which could have a dual effect on complement resistance. As a net effect the degradation of serum complement proteins by PgtE seems beneficial for Salmonella survival in the host as it resulted in decreased opsonization and phagocytosis.

S. enterica spreads in the host by repeated cycles of phagocyte infection (Mastroeni et al., 2009). Within the phagocytes the activity of PgtE is high, in part due to decreased expression of the $\mathrm{O}$-antigen that inhibits substrate binding by PgtE (Lahteenmaki et al., 2005). On the other hand, O-antigen is a major defense structure of Salmonella against complement killing (Frank et al., 1987; Delgado et al., 2006). Our hypothesis is that degradation of $\mathrm{B}$ and $\mathrm{H}$ by PgtE contributes to the survival of $S$. enterica, when the bacterium is released from one phagocyte and infects another one.

To our knowledge, the only microbial protease that has been reported to cleave factor B to date is an unidentified secreted protease of Leptospira spp (Fraga et al., 2014). Factor B circulates in blood as a proenzyme with the scissile bond Arg234-Lys235 between $\mathrm{Ba}$ and $\mathrm{Bb}$ being protected against factor $\mathrm{D}$-mediated activation. Because the von Willebrand factor type A domain in $\mathrm{Bb}$ and the preceding SCR domains in $\mathrm{Ba}$ are all involved in binding of $\mathrm{B}$ to $\mathrm{C} 3 \mathrm{~b}$, and given that $\mathrm{Bb}$, once dissociated from $\mathrm{C} 3 \mathrm{bBb}$ convertase, is unable to reassociate with $\mathrm{C} 3 \mathrm{~b}$, it is likely that PgtEcleaved $\mathrm{B}$ cannot associate with $\mathrm{C} 3 \mathrm{~b}$ to form a C3-convertase (Milder et al., 2007; Torreira et al., 2009).

Previously, an unidentified secreted protease of Aspergillus fumigatus and the serine protease dentilisin of the periodontal pathogen Treponema denticola have been reported to cleave H (McDowell et al., 2009; Behnsen et al., 2010). Recruitment of $\mathrm{H}$ by $\mathrm{FhbB}$, the $\mathrm{H}$ binding protein of $T$. denticola, facilitates the dentilisin-mediated cleavage (McDowell et al., 2009; Miller et al., 2012). In Salmonella, the expression of the H binding protein, Rck,requires heterologous quorum sensing signals (Michael et al., 2001; Ho et al., 2010) and may thereby contribute to PgtE-mediated cleavage.

Factor $\mathrm{H}$ is a single-chain polypeptide that folds into 20 globular SCR domains connected by short linkers. Given that the intra-SCR thioester bonds were insufficient to maintain the beads-on-a-string configuration after cleavage by Pla or PgtE, the cleavage of $\mathrm{H}$ most probably occurred within the linkers. The proportion of Arg and Lys residues that are preferentially targeted in omptin proteolysis (Hritonenko and Stathopoulos, 2007) is higher in $\mathrm{H}$ linkers than in the human proteome on average: 1.7and 2.6-fold higher, respectively (Lehtinen et al., 2004). This may render the linker regions candidate targets for cleavage by PgtE and Pla. Both PgtE and Pla cleaved H N-terminally to SCR7 suggesting that also FHL-1, the alternatively spliced product of the human CFH gene containing H SCRs 1-7 (Estaller et al., 1991) could be cleaved by these omptins.

What could be the functional consequence of the proteolytic attack on H? Cleavage within SCRs $1-5$ by PgtE and shortly after SCR5 by Pla separates the cofactor activity located within SCRs 1-4 and the polyanion and microbial binding sites within SCRs 19-20 and 6-7. Thus, the omptin-cleaved $\mathrm{H}$ cannot be targeted onto a microbial or host surface to regulate complement activation. Cleavage by PgtE most probably destroys also the cofactor activity itself since SCR domains 1 through 4 are all needed to mediate the cofactor activity in $\mathrm{H}$ (Kühn et al., 1995; Makou et al., 2013; Meri et al., 2013). This could lead to more fulminant pathology since the $\mathrm{H}$-mediated protection of host structures would also be compromised. Importantly, the resulting inability of PgtE-cleaved $\mathrm{H}$ to control complement activation in the fluid phase would accelerate the depletion of C3 seen with PgtEexpressing Salmonella. The local depletion of C3 would efficiently block complement attack against the bacteria.

Although PgtE cleaves complement effector molecules, it has been reported to mediate only modest resistance to direct bactericidal activity of serum (Ramu et al., 2007). Also for Y. pestis, Pla is dispensable for serum resistance despite C3 cleavage (Sodeinde et al., 1992; Bartra et al., 2008). Instead, C3 cleavage and interference of complement activation by these omptins could affect phagocytosis since $\mathrm{C} 3 \mathrm{~b}$ and $\mathrm{iC} 3 \mathrm{~b}$ are key opsonins. Interestingly, van Bruggen and co-workers showed that the $\mathrm{iC} 3 \mathrm{~b}$ receptor CR3 (CD11b/CD18) mediated the internalization of S. enterica 14028 into human neutrophils (van Bruggen et al., 2007). Neutrophils kill Salmonella more efficiently than macrophages (Burton et al., 2014). Here we showed that PgtE expression impairs the interaction, either the adherence or the uptake or both, between Salmonella and human neutrophils. We postulate that this is due to PgtE-dependent breakdown of C3 into fragments that have no opsonizing capacity. Importantly, the PgtE-mediated cleavage of $\mathrm{C} 3, \mathrm{H}$, and $\mathrm{B}$ can contribute at several branches in the complement activation cascade to the diminished formation of $\mathrm{iC} 3 \mathrm{~b}$, the preferred ligand for CR3, as illustrated in Figure 6B.

In conclusion, this work showed that PgtE cleaves factors B and $\mathrm{H}$ thus targeting both the activating and the inhibitory arms of complement. The overall effect of PgtE favors protection of Salmonella against the human host immune system, since C3 became depleted from serum, less C3-derived fragments accumulated on Salmonella and the association with neutrophils was reduced along with PgtE expression. The results thus suggest that PgtE has an important role in Salmonella pathogenesis.

\section{MATERIALS AND METHODS BACTERIAL STRAINS AND PLASMIDS}

The previously characterized bacteria and plasmids are listed in Table 1. E. coli XL1 - strains were grown, and omptin expression was induced by $5 \mu \mathrm{M}$ IPTG as previously described (Kukkonen et al., 2001). Other strains were grown with shaking at $37^{\circ} \mathrm{C}$ overnight, E. coli $83972 \Delta o m p T$ strains and plasmid-complemented Salmonella 14028R-1 strains in LuriaBertani containing $100 \mu \mathrm{g} / \mathrm{ml}$ ampicillin and $5 \mu \mathrm{M}$ IPTG, and Salmonella strains 14028R and 14028R-1 in pgtE-inducing media (Lahteenmaki et al., 2005). For C3 cleavage assay the plasmidcomplemented Salmonella strains were diluted 1:50 with fresh broth in the morning and grown for $2 \mathrm{~h}$ further. Bacteria were harvested by centrifugation and washed three times with PBS, $\mathrm{pH} 7.4-7.5$, or veronal buffered saline $(142 \mathrm{mM} \mathrm{NaCl}, 1.8 \mathrm{mM}$ Na-barbital, $3.3 \mathrm{mM}$ barbituric acid, pH 7.4) (VBS).

\section{SERA, PLASMA AND PMN}

Peripheral blood for PMN isolation, for a pool of normal human serum (NHS) and for a pool of plasma for C3 isolation was 
Table 1 | Bacterial strains and plasmids.

\begin{tabular}{|c|c|c|}
\hline Strain or plasmid & Description & $\begin{array}{l}\text { Source or } \\
\text { references }\end{array}$ \\
\hline \multicolumn{3}{|l|}{ ESCHERICHIA COLI } \\
\hline $83972 \Delta$ ompT & $\Delta o m p T$ derivative of 83972 & Järvinen et al., 2013 \\
\hline XL1 Blue MRF' & $\begin{array}{l}\Delta \text { (mcrA)183 } \\
\Delta \text { (mcrCB-hsdSMR-mrr)173 } \\
\text { endA1 supE44 thi-1 recA1 } \\
\text { gyrA96 relA1 lac [F ì proAB } \\
\left.\text { lac/9 Z } \triangle 15 \text { Tn } 10\left(\text { Tet }^{r}\right)\right]\end{array}$ & Agilent Technologies \\
\hline \multicolumn{3}{|c|}{ SALMONELLA ENTERICA SEROVAR TYPHIMURIUM } \\
\hline $14028 \mathrm{R}$ & rough LPS derivative of 14028 & Wick et al., 1994 \\
\hline 14028R-1 & $\Delta p g t E$ derivative of $14028 \mathrm{R}$ & $\begin{array}{l}\text { Lahteenmaki et al., } \\
2005\end{array}$ \\
\hline \multicolumn{3}{|l|}{ PLASMID } \\
\hline pSE380 & $\begin{array}{l}\text { expression vector, trc } \\
\text { promoter, lacO operator, lacl, } \\
\text { bla }\end{array}$ & $\begin{array}{l}\text { Thermo Fischer } \\
\text { Scientific Inc. }\end{array}$ \\
\hline pMRK1 & pla (Y. pestis) in pSE380 & Kukkonen et al., 2001 \\
\hline pMRK1206 & pla D206A in pSE380 & Kukkonen et al., 2001 \\
\hline pMRK2 & ompT (E. coli) in pSE380 & Kukkonen et al., 2001 \\
\hline pMRK3 & pgtE (S. enterica) in pSE380 & $\begin{array}{l}\text { Kukkonen et al., } \\
2004\end{array}$ \\
\hline pMRK31 & pgtE D206A in pSE380 & $\begin{array}{l}\text { Kukkonen et al., } \\
2004\end{array}$ \\
\hline pMRK6 & $\operatorname{omp} P(E$. coli) in pSE380 & Järvinen et al., 2013 \\
\hline pMRK8 & $y c o A(Y$. pestis $)$ in pSE380 & Haiko et al., 2010 \\
\hline pMRK9 & $\begin{array}{l}\text { ycoB (Y. pseudotuberculosis) } \\
\text { in pSE380 }\end{array}$ & Haiko et al., 2010 \\
\hline
\end{tabular}

drawn from consented healthy laboratory personnel. Pooled NHS was aliquoted into single-use batches and stored at $-72^{\circ} \mathrm{C}$. The NHS pool tested negative for diagnostic Salmonella antibodies in $\mathrm{O}-$ and $\mathrm{H}$-antigen agglutination tests and in ELISA measuring IgA, IgG, and IgM against LPS of Salmonella Typhimurium and Enteritidis. To prepare heat-inactivated serum (HIS), NHS was incubated for $30 \mathrm{~min}$ at $56^{\circ} \mathrm{C}$. PMN were isolated by Ficoll-Paque PLUS centrifugation as in Jarva et al. (2002) and resuspended in $0.1 \%$ BSA-VBS for the phagocytosis assay.

\section{COMPLEMENT PROTEINS AND ANTIBODIES}

Factor D was purchased from Merck Millipore. Factors $\mathrm{H}$ and B were from Merck Millipore and Quidel Corp. C3 was purified from plasma (Koistinen et al., 1989). H fragments SCR1-5 and SCR15-20 were produced as His-tagged proteins in the baculovirus expression system and purified with Ni-Sepharose (Kühn and Zipfel, 1995). Rabbit antisera against C3c and C3d were from Dako. Goat antisera against B and $\mathrm{H}$ were from Merck Millipore.

Monoclonal antibodies 131X, 196X, and 3D11 against $\mathrm{H}$ have been described previously (Fontaine et al., 1989; Jokiranta et al., 1996). Penta-His mAb was from Qiagen. HRP-conjugated secondary antibodies were from Jackson ImmunoResearch Laboratories.

\section{CLEAVAGE OF COMPLEMENT PROTEINS}

Two parts of bacterial suspension (OD600 $=2$ in PBS or VBS) were mixed with one part of protein solution (for $\mathrm{H}$, stock
$100 \mu \mathrm{g} / \mathrm{ml}$; B, 100 or $50 \mu \mathrm{g} / \mathrm{ml}$; SCR $1-5,25 \mu \mathrm{g} / \mathrm{ml}$; SCR15-20, $30 \mu \mathrm{g} / \mathrm{ml})$. Samples were incubated with shaking at $37^{\circ} \mathrm{C}$ for the indicated times. Bacteria were pelleted at 10,000 $\mathrm{g}$ for $3 \mathrm{~min}$. Control cleavage of $\mathrm{B}$ by factor $\mathrm{D}$ was performed as follows: $\mathrm{B}$ $(0.5 \mu \mathrm{g})$ was incubated with $\mathrm{D}(0.025 \mu \mathrm{g})$ and $\mathrm{C} 3(1 \mu \mathrm{g})$ in $15 \mu \mathrm{l}$ of $5 \mathrm{mM} \mathrm{MgCl} 2$-VBS for $1 \mathrm{~h}$. The supernatants (3 parts) were mixed with 1 part of either reducing or non-reducing $4 \mathrm{x}$ Laemmli sample buffer (LSB) and heated at $95^{\circ} \mathrm{C}$ for $3-5 \mathrm{~min}$ followed by SDS-PAGE and immunoblotting.

\section{SDS-PAGE AND WESTERN BLOTTING}

The recombinant $\mathrm{H}$ fragments were resolved in 15\% SDSPAGE. For the other samples $10 \%$ gels were used. Proteins were transferred onto a $0.2 \mu \mathrm{m}$ nitrocellulose membrane (BioRad Laboratories). The blocking solution and antibody diluent was $5 \%$ skimmed milk/PBS. Enhanced chemiluminescence was detected on Super-RX films (Fujifilm Corporation).

\section{C3 CLEAVAgE IN SERUM}

Salmonella bacteria $\left(2 \times 10^{8} \mathrm{CFU} / \mathrm{ml}\right)$ were incubated in $5 \%$ NHS/VBS for $1 \mathrm{~h}$ at $37^{\circ} \mathrm{C}$ with shaking. The bacteria were pelleted, and three parts of the supernatant were mixed with one part of reducing $4 \mathrm{x}$ LSB. The pellets were washed with VBS and resuspended in reducing $1 \mathrm{x}$ LSB to the original reaction volume. The samples were heated at $95^{\circ} \mathrm{C}$ for $5 \mathrm{~min}$ and run in $10 \%$ SDS-PAGE followed by immunoblotting with anti-C3c and C3d antibodies.

\section{OPSONOPHAGOCYTOSIS ASSAY}

The phagocytosis protocol was modified from Jarva et al. (2002). Briefly, Salmonella strains were grown overnight in pgtE-inducing medium and labeled with FITC-Celite ${ }^{\circledR}$ (Sigma-Aldrich) by incubating them for $15 \mathrm{~min}$ with shaking at $37^{\circ} \mathrm{C}$ in $0.5 \mu \mathrm{g} / \mu \mathrm{l}$ FITCCelite ${ }^{\circledR} / \mathrm{PBS}$ in the dark. The bacteria were washed three times and adjusted to an OD600 value of 0.2 in $0.1 \%$ BSA-VBS. Thereafter, the bacteria were incubated in $50 \%$ NHS, $50 \%$ HIS or plain buffer for $5 \mathrm{~min}$ with agitation at $37^{\circ} \mathrm{C}$. Freshly isolated $\mathrm{PMN}$ were added at a MOI of 20. The PMN were fixed after 5 and $10 \mathrm{~min}$ in $2 \%$ PFA/PBS for 15 min and run in PBS on FACScan (Becton, Dickinson and Company). The FITC signal was measured from 10,000 PMN and data was analyzed in Summit v4.3 (Beckman Coulter). A comparable FITC-staining of Salmonella strains was confirmed using flow cytometry.

\section{AUTHOR CONTRIBUTIONS}

Rauna Riva and Seppo Meri devised the experiments, Rauna Riva conducted the experiments, Rauna Riva, Seppo Meri, and Timo K. Korhonen formulated the manuscript.

\section{ACKNOWLEDGMENTS}

This work was supported by the Magnus Ehrnrooth Foundation, the Sigrid Jusélius Foundation, the state competitive research funding grants TYH2012237 and TYH2014307, and the Stockmann Foundation. We thank Hanna Järvinen for the production of recombinant $\mathrm{H}$ fragments, Vesa Koistinen for the purification of C3, and Laura Degerstedt, Marjatta Ahonen, Pirkko Kokkonen, and Kirsti Widing for their skilled technical assistance. 


\section{REFERENCES}

Bajic, G., Yatime, L., Sim, R. B., Vorup-Jensen, T., and Andersen, G. R. (2013). Structural insight on the recognition of surface-bound opsonins by the integrin I domain of complement receptor 3. Proc. Natl. Acad. Sci. U.S.A. 110, 16426-16431. doi: 10.1073/pnas.1311261110

Barthel, D., Schindler, S., and Zipfel, P. F. (2012). Plasminogen is a complement inhibitor. J. Biol. Chem. 287, 18831-18842. doi: 10.1074/jbc.M111.323287

Bartra, S. S., Styer, K. L., O’Bryant, D. M., Nilles, M. L., Hinnebusch, B. J., Aballay, A., et al. (2008). Resistance of Yersinia pestis to complement-dependent killing is mediated by the Ail outer membrane protein. Infect. Immun. 76, 612-622. doi: 10.1128/IAI.01125-07

Behnsen, J., Lessing, F., Schindler, S., Wartenberg, D., Jacobsen, I. D., Thoen, M., et al. (2010). Secreted Aspergillus fumigatus protease Alp1 degrades human complement proteins C3, C4, and C5. Infect. Immun. 78, 3585-3594. doi: 10.1128/IAI.01353-09

Blom, A. M., Hallström, T., and Riesbeck, K. (2009). Complement evasion strategies of pathogens-Acquisition of inhibitors and beyond. Mol. Immunol. 46, 2808-2817. doi: 10.1016/j.molimm.2009.04.025

Buckle, G. C., Walker, C. L., and Black, R. E. (2010). Typhoid fever and paratyphoid fever: systematic review to estimate global morbidity and mortality for. J. Glob. Health 2:010401. doi: 10.7189/jogh.02.010401

Burton, N. A., Schurmann, N., Casse, O., Steeb, A. K., Claudi, B., Zankl, J., et al. (2014). Disparate impact of oxidative host defenses determines the fate of Salmonella during systemic infection in mice. Cell Host Microbe 15, 72-83. doi: 10.1016/j.chom.2013.12.006

Crump, J. A., Luby, S. P., and Mintz, E. D. (2004). The global burden of typhoid fever. Bull. World Health Organ. 82, 346-353.

Delgado, M. A., Mouslim, C., and Groisman, E. A. (2006). The PmrA/PmrB and RcsC/YojN/RcsB systems control expression of the Salmonella O-antigen chain length determinant. Mol. Microbiol. 60, 39-50. doi: 10.1111/j.13652958.2006.05069.x

Eren, E., and van den Berg, B. (2012). Structural basis for activation of an integral membrane protease by lipopolysaccharide. J. Biol. Chem. 287, 23971-23976. doi: 10.1074/jbc.M112.376418

Eren, E., Murphy, M., Goguen, J., and van den Berg, B. (2010). An active site water network in the plasminogen activator Pla from Yersinia pestis. Structure 18, 809-818. doi: 10.1016/j.str.2010.03.013

Eriksson, S., Lucchini, S., Thompson, A., Rhen, M., and Hinton, J. C. (2003). Unravelling the biology of macrophage infection by gene expression profiling of intracellular Salmonella enterica. Mol. Microbiol. 47, 103-118. doi: 10.1046/j.1365-2958.2003.03313.x

Estaller, C., Weiss, E. H., Schwaeble, W., and Dierich, M. (1991). Human complement factor $\mathrm{H}$ : two factor $\mathrm{H}$ proteins are derived from alternatively spliced transcripts. Eur. J. Immunol. 21, 799-802. doi: 10.1002/eji.1830210337

Eurosurveillance Editorial Team. (2012). The European Union summary report on trends and sources of zoonoses, zoonotic agents and food-borne outbreaks in. EFSA J. 12:3547. doi: 10.2903/j.efsa.2014.3547

Fontaine, M., Demares, M. J., Koistinen, V., Day, A. J., Davrinche, C., Sim, R. B., et al. (1989). Truncated forms of human complement factor H. Biochem. J. 258, 927-930.

Fraga, T. R., Courrol Ddos, S., Castiblanco-Valencia, M. M., Hirata, I. Y., Vasconcellos, S. A., et al. (2014). Immune evasion by pathogenic Leptospira strains: the secretion of proteases that directly cleave complement proteins. J. Infect. Dis. 209, 876-886. doi: 10.1093/infdis/jit569

Franco, A. A., Kothary, M. H., Gopinath, G., Jarvis, K. G., Grim, C. J., and Hu, L. et al. (2011). Cpa, the outer membrane protease of Cronobacter sakazakii, activates plasminogen and mediates resistance to serum bactericidal activity. Infect. Immun. 79, 1578-1587. doi: 10.1128/IAI.01165-10

Frank, M. M., Joiner, K., and Hammer, C. (1987). The function of antibody and complement in the lysis of bacteria. Rev. Infect. Dis. 9(Suppl. 5), S537-S45.

Graham, S. M. (2010). Nontyphoidal salmonellosis in Africa. Curr. Opin. Infect. Dis. 23, 409-414. doi: 10.1097/QCO.0b013e32833dd25d

Haiko, J., Laakkonen, L., Juuti, K., Kalkkinen, N., and Korhonen, T. K. (2010). The omptins of Yersinia pestis and Salmonella enterica cleave the reactive center loop of plasminogen activator inhibitor 1. J. Bacteriol. 192, 4553-4561. doi: 10.1128/JB.00458-10

Ho, D. K., Jarva, H., and Meri, S. (2010). Human complement factor H binds to outer membrane protein Rck of Salmonella. J. Immunol. 185, 1763-1769. doi: 10.4049/jimmunol.1001244
Hritonenko, V., and Stathopoulos, C. (2007). Omptin proteins: an expanding family of outer membrane proteases in Gram-negative Enterobacteriaceae. Mol. Membr. Biol. 24, 395-406. doi: 10.1080/09687680701443822

Jarva, H., Janulczyk, R., Hellwage, J., Zipfel, P. F., Björck, L., and Meri, S. (2002). Streptococcus pneumoniae evades complement attack and opsonophagocytosis by expressing the $p s p C$ locus-encoded Hic protein that binds to short consensus repeats 8-11 of factor H. J. Immunol. 168, 1886-1894. doi: 10.4049/jimmunol.168.4.1886

Järvinen, H. M., Laakkonen, L., Haiko, J., Johansson, T., Juuti, K., and Suomalainen, M. et al. (2013). Human single-chain urokinase is activated by the omptins PgtE of Salmonella enterica and Pla of Yersinia pestis despite mutations of active site residues. Mol. Microbiol. 89, 507-517. doi: 10.1111/mmi.12293

Jokiranta, T. S., Zipfel, P. F., Hakulinen, J., Kuhn, S., Pangburn, M. K., Tamerius, J. D., et al. (1996). Analysis of the recognition mechanism of the alternative pathway of complement by monoclonal anti-factor $\mathrm{H}$ antibodies: evidence for multiple interactions between $\mathrm{H}$ and surface bound C3b. FEBS Lett. 393, 297-302. doi: 10.1016/0014-5793(96)00905-2

Koistinen, V., Wessberg, S., and Leikola, J. (1989). Common binding region of complement factors $\mathrm{B}, \mathrm{H}$ and $\mathrm{CR} 1$ on $\mathrm{C} 3 \mathrm{~b}$ revealed by monoclonal anti-C3d. Complement Inflamm. 6, 270-280.

Korhonen, T. K., Haiko, J., Laakkonen, L., Jarvinen, H. M., and WesterlundWikstrom, B. (2013). Fibrinolytic and coagulative activities of Yersinia pestis. Front. Cell. Infect. Microbiol. 3:35. doi: 10.3389/fcimb.2013.00035

Kühn, S., and Zipfel, P. F. (1995). The baculovirus expression vector pBSV-8His directs secretion of histidine-tagged proteins. Gene 162, 225-229.

Kühn, S., Skerka, C., and Zipfel, P. F. (1995). Mapping of the complement regulatory domains in the human factor $\mathrm{H}$-like protein 1 and in factor H1. J. Immunol. 155, 5663-5670.

Kukkonen, M., Lahteenmaki, K., Suomalainen, M., Kalkkinen, N., Emody, L., Lang, H., et al. (2001). Protein regions important for plasminogen activation and inactivation of $\alpha 2$-antiplasmin in the surface protease Pla of Yersinia pestis. Mol. Microbiol. 40, 1097-1111. doi: 10.1046/j.1365-2958.2001.02451.x

Kukkonen, M., Suomalainen, M., Kyllonen, P., Lahteenmaki, K., Lang, H., and Virkola, R. et al. (2004). Lack of O-antigen is essential for plasminogen activation by Yersinia pestis and Salmonella enterica. Mol. Microbiol. 51, 215-225. doi: 10.1046/j.1365-2958.2003.03817.x

Lahteenmaki, K., Kyllonen, P., Partanen, L., and Korhonen, T. K. (2005). Antiprotease inactivation by Salmonella enterica released from infected macrophages. Cell. Microbiol. 7, 529-538. doi: 10.1111/j.1462-5822.2004.00483.x

Lathem, W. W., Price, P. A., Miller, V. L., and Goldman, W. E. (2007). A plasminogen-activating protease specifically controls the development of primary pneumonic plague. Science 315, 509-513. doi: 10.1126/science.1137195

Lehtinen, M. J., Meri, S., and Jokiranta, T. S. (2004). Interdomain contact regions and angles between adjacent short consensus repeat domains. J. Mol. Biol. 344, 1385-1396. doi: 10.1016/j.jmb.2004.10.017

Makou, E., Herbert, A. P., and Barlow, P. N. (2013). Functional anatomy of complement factor H. Biochemistry 52, 3949-3962. doi: 10.1021/bi4003452

Mastroeni, P., Grant, A., Restif, O., and Maskell, D. (2009). A dynamic view of the spread and intracellular distribution of Salmonella enterica. Nat. Rev. Microbiol. 7, 73-80. doi: 10.1038/nrmicro2034

McDowell, J. V., Huang, B., Fenno, J. C., and Marconi, R. T. (2009). Analysis of a unique interaction between the complement regulatory protein factor $\mathrm{H}$ and the periodontal pathogen Treponema denticola. Infect. Immun. 77, 1417-1425. doi: 10.1128/IAI.01544-08

Meri, T., Amdahl, H., Lehtinen, M. J., Hyvarinen, S., McDowell, J. V., and Bhattacharjee, A. et al. (2013). Microbes bind complement inhibitor factor $\mathrm{H}$ via a common site. PLoS Pathog. 9:e1003308. doi: 10.1371/journal.ppat.1003308

Michael, B., Smith, J. N., Swift, S., Heffron, F., and Ahmer, B. M. M. (2001). SdiA of Salmonella enterica is a LuxR homolog that detects mixed microbial communities. J. Bacteriol. 183, 5733-5742. doi: 10.1128/JB.183.19.5733-5742.2001

Milder, F. J., Gomes, L., Schouten, A., Janssen, B. J., Huizinga, E. G., and Romijn, R. A. et al. (2007). Factor B structure provides insights into activation of the central protease of the complement system. Nat. Struct. Mol. Biol. 14, 224-228. doi: $10.1038 / \mathrm{nsmb} 1210$

Miller, D. P., Bell, J. K., McDowell, J. V., Conrad, D. H., Burgner, J. W., Héroux, A., et al. (2012). Structure of factor H-binding protein B (FhbB) of the periopathogen, Treponema denticola: Insights into progression of periodontal disease. J. Biol. Chem. 287, 12715-12722. doi: 10.1074/jbc.M112.339721 
Montminy, S. W., Khan, N., McGrath, S., Walkowicz, M. J., Sharp, F., and Conlon, J. E. et al. (2006). Virulence factors of Yersinia pestis are overcome by a strong lipopolysaccharide response. Nat. Immunol. 7, 1066-1073. doi: 10.1038/ni1386

Podack, E. R., and Muller-Eberhard, H. J. (1979). Isolation of human S-protein, an inhibitor of the membrane attack complex of complement. J. Biol. Chem. 254, 9808-9814.

Ramu, P., Lobo, L. A., Kukkonen, M., Bjur, E., Suomalainen, M., and Raukola, H. et al. (2008). Activation of pro-matrix metalloproteinase-9 and degradation of gelatin by the surface protease PgtE of Salmonella enterica serovar Typhimurium. Int. J. Med. Microbiol. 298, 263-278. doi: 10.1016/j.ijmm.2007.06.004

Ramu, P., Tanskanen, R., Holmberg, M., Lahteenmaki, K., Korhonen, T. K., and Meri, S. (2007). The surface protease PgtE of Salmonella enterica affects complement activity by proteolytically cleaving C3b, C4b and C5. FEBS Lett. 581, 1716-1720. doi: 10.1016/j.febslet.2007.03.049

Ricklin, D., Hajishengallis, G., Yang, K., and Lambris, J. D. (2010). Complement: a key system for immune surveillance and homeostasis. Nat. Immunol. 11, 785-797. doi: 10.1038/ni.1923

Sebbane, F., Jarrett, C. O., Gardner, D., Long, D., and Hinnebusch, B. J. (2006). Role of the Yersinia pestis plasminogen activator in the incidence of distinct septicemic and bubonic forms of flea-borne plague. Proc. Natl. Acad. Sci. U.S.A. 103, 5526-5530. doi: 10.1073/pnas.0509544103

Skurnik, M., Peippo, A., and Ervela, E. (2000). Characterization of the O-antigen gene clusters of Yersinia pseudotuberculosis and the cryptic $\mathrm{O}$-antigen gene cluster of Yersinia pestis shows that the plague bacillus is most closely related to and has evolved from Y. pseudotuberculosis serotype O:1b. Mol. Microbiol. 37, 316-330. doi: 10.1046/j.1365-2958.2000.01993.x

Sodeinde, O. A., Subrahmanyam, Y. V., Stark, K., Quan, T., Bao, Y., and Goguen, J. D. (1992). A surface protease and the invasive character of plague. Science 258, 1004-1007.

Suomalainen, M., Lobo, L. A., Brandenburg, K., Lindner, B., Virkola, R., and Knirel, Y. A. et al. (2010). Temperature-induced changes in the lipopolysaccharide of Yersinia pestis affect plasminogen activation by the Pla surface protease. Infect. Immun. 78, 2644-2652. doi: 10.1128/IAI.01329-09
Torreira, E., Tortajada, A., Montes, T., Rodriguez de Cordoba, S., and Llorca, O. (2009). 3D structure of the C3bB complex provides insights into the activation and regulation of the complement alternative pathway convertase. Proc. Natl. Acad. Sci. U.S.A. 106, 882-887. doi: 10.1073/pnas.08108 60106

van Bruggen, R., Zweers, D., van Diepen, A., van Dissel, J. T., Roos, D., Verhoeven, A. J., et al. (2007). Complement receptor 3 and Toll-like receptor 4 act sequentially in uptake and intracellular killing of unopsonized Salmonella enterica serovar Typhimurium by human neutrophils. Infect. Immun. 75, 2655-2660. doi: 10.1128/IAI.01111-06

Wick, M. J. (2011). Innate immune control of Salmonella enterica serovar Typhimurium: Mechanisms contributing to combating systemic Salmonella infection. J. Innate Immun. 3, 543-549. doi: 10.1159/000330771

Wick, M. J., Harding, C. V., Normark, S. J., and Pfeifer, J. D. (1994). Parameters that influence the efficiency of processing antigenic epitopes expressed in Salmonella typhimurium. Infect. Immun. 62, 4542-4548.

Conflict of Interest Statement: The authors declare that the research was conducted in the absence of any commercial or financial relationships that could be construed as a potential conflict of interest.

Received: 25 November 2014; accepted: 18 January 2015; published online: 06 February 2015.

Citation: Riva R, Korhonen TK and Meri S (2015) The outer membrane protease PgtE of Salmonella enterica interferes with the alternative complement pathway by cleaving factors B and H. Front. Microbiol. 6:63. doi: 10.3389/fmicb.2015.00063

This article was submitted to Microbial Immunology, a section of the journal Frontiers in Microbiology.

Copyright (C) 2015 Riva, Korhonen and Meri. This is an open-access article distributed under the terms of the Creative Commons Attribution License (CC BY). The use, distribution or reproduction in other forums is permitted, provided the original author(s) or licensor are credited and that the original publication in this journal is cited, in accordance with accepted academic practice. No use, distribution or reproduction is permitted which does not comply with these terms. 